\title{
Iron-dextran as a thermosensitizer in radiofrequency hyperthermia for cancer treatment
}

\author{
Hea-Jong Chung ${ }^{1}$, Hyeon-Jin Kim² ${ }^{2}$ and Seong-Tshool Hong ${ }^{1 *}$
}

\begin{abstract}
Radiofrequency hyperthermia is a recently rediscovered oncotherapy rising in popularity. However, lack of a proper thermosensitizer limits current radiofrequency hyperthermia to be only slightly effective, mostly being used as a subsidiary to a standard oncotherapy. Here, we report that iron-dextran delivers iron ion to cancer cells for cancer-selective accumulation of the iron ion, which functions as a thermosensitizer for radiofrequency hyperthermia. Intravenous injection of iron-dextran to tumor-xenografted mice resulted in selective accumulation of iron ion in the targeted cancer cells. The accumulated iron ion in cancer cells dramatically reacted to radiofrequency wave to result in tumorselective dielectric temperature increment without harming the surrounding normal tissue. The oncotherapeutic effect of was evaluated using tumor-xenografted mice. The overall anticancer efficacy of radiofrequency hyperthermia after injection of iron-dextran as a thermosensitizer in breast cancer-bearing mice was much better than the efficacy of paclitaxel, a standard chemotherapy drug for cancer. Moreover, hyperthermia using iron-dextran as a thermosensitizer completely eradicated cancer in the tumor xenografted mice. This work suggests that iron-dextran is an ideal thermosensitizer for radiofrequency hyperthermia. We believe that the application of iron-dextran as a thermosensitizer would be a major progress in hyperthermia cancer treatments.
\end{abstract}

Keywords: Cancer, Hyperthermia, Iron-dextran, Radiofrequency, Thermosensitizer

\section{Introduction}

Treatment of cancer by temperature elevation has been developed from ancient time to modern medicine. The thermal cancer treatment in modern medicine is achieved by ablation and hyperthermia. Ablation uses high heat, typically $>46{ }^{\circ} \mathrm{C}$, to burn cancer tissue, in which laser lights are used. While, hyperthermia uses moderate temperature ranging from 39 to $46{ }^{\circ} \mathrm{C}$. The purpose of hyperthermia is to increase temperature of interbody cancer to induce death of cancer cells either by apoptosis, necrosis or both.

One of the key characteristics of cancer cells is uncontrolled cell division. The uncontrolled cell division of

\footnotetext{
*Correspondence: seonghong@chonbuk.ac.kr

${ }^{1}$ Department of Biomedical Sciences and Institute for Medical Science, Chonbuk National University Medical School, Jeonju, Chonbuk, South Korea

Full list of author information is available at the end of the article
}

cancer cells leads to the formation of an unorganized piling of cell mass which makes it impossible to properly develop neovascularization as in the case of normal tissues of the body [1]. Thus, cancer tissues are limited of blood circulation, leading to a constant hypoxic condition where glycolysis is facilitated to acidify the environment [2]. In addition to excessive glycolysis, poor blood circulation also makes cancer tissues vulnerable to high temperature. Unlike normal tissues, the excessive heat in the tumor tissues is difficult to be diffused into other parts of body via blood flow [3]. Based on such characteristics of cancer tissues, hyperthermic oncotherapies, in which cancer tissue is heated to high temperatures, were developed (Fig. 2a, b). Although the concept of hyperthermic oncotherapies was developed several decades ago, the therapeutic efficacy and benefit of hyperthermic oncotherapies have not noticed until recently [4]. Hyperthermic oncotherapies have reasonable therapeutic efficacy with almost no side effect so as to be getting 
popularity currently. Hyperthermic oncotherapies especially in combination with radiotherapy showed excellent oncotherapeutic efficacy.

Elevation of the temperature in cancer can be achieved by various methods such as radiofrequency (RF), vibration of metal nanoparticles in alternative magnetic field, metal nanoparticle in an electromagnetic wave, pyrogenic chemicals, etc. Among these hyperthermal methods, magnetic or metal nanoparticles has got an attention in hyperthermal community and thus most thoroughly investigated so that some of these magnetic or metal nanoparticles even investigated in clinical trials [5]. Although magnetic or metal nanoparticles showed some efficacy, the insoluble nature of the magnetic or metal nanoparticles raised some concern [6].

Metal ions are known to interact well with electromagnetic waves [7]. Especially, interaction of metal ions which has a very strong dipole moment such as iron ion generates strong dielectric heat when radiated by RF wave [8]. Because of this characteristic, iron ion could be an excellent thermosensitizer in RF thermotherapy if iron ion can be delivered specifically to cancer cells. Considering the fact that X-ray and red light requires sensitizers for an effective oncotherapy, it would be reasonable to speculate that the efficacy of RF thermotherapy could be significantly improved when paired with a sensitizing reagent (Additional file 1: Table S1). To function as a tumor-targeting thermosensitizer, iron ion has to be accumulated more selectively in cancer cells than in normal cells. Iron-dextran, a medication for anemia, is a complex material in which iron ion form a complex with dextran through intermolecular bonding [9]. Because dextran has very strong tumor-targeting characteristics [10], we speculated whether the injection of iron-dextran would result in accumulation of iron ion in cancer cells. If iron-dextran is able to selectively deliver iron ion to cancer cells, iron-dextran would be an ideal thermosensitizer for RF thermotherapy. In this work, we showed that irondextran specifically delivered iron ion to cancer cells and thereby selectively increased the temperature of cancer tissue upon RF hyperthermic treatment. Our results confirmed successful eradication of cancer in a RF thermotherapy of tumor-xenografted animals when iron-dextran was used as a thermosensitizer, making iron-dextran a novel thermosensitizer candidate for RF thermotherapy to cure cancer.

\section{Materials and methods}

\section{Cell lines and cell culture}

The cell lines and cell culture protocol of this study using were followed as previously described [11]. The detailed protocol for culture is described Additional file 1.

\section{Animal study condition}

The protocol was approved by the Ethics Committee of Chonbuk National University Laboratory Animal Center (Permit Number: CBU 2012-0040) in accordance with the 'Guide for the Care and Use of Laboratory Animals', published by the National Research Council and endorsed by the ARRIVE Guidelines. The animal care protocol was followed as previously described [11-14].

\section{Study of generating heat by iron-dextran in vitro}

To evaluate the thermal performance of dextran and iron-dextran (Sigma-Aldrich, St. Louis, Missouri, USA), $0.1 \mathrm{~mL}$ of $5 \mathrm{mg} / \mathrm{mL}$ dextran or iron-dextran solutions were added onto 96-well plates in triplicate. After that, it was exposed for $10 \mathrm{~min}$ by the electromagnetic wavedependent hyperthermia (LAB-EHY 100; Oncotherm, Budaörs, Hungary) at $50 \mathrm{~W}$ energy dose. The evaluation of heat capacity of solutions used by the thermal imaging camera (FLIR-E60; FLIR, Wilsonville, Oregon, USA) and the temperature of solutions measured by DirA Program (FLIR Tools; FLIR, Wilsonville, Oregon, USA). The iron-dextran in vitro condition protocol was followed as previously described [11]. The detailed protocol for iron-dextran in vitro condition is described in the Additional file 1. All data are presented as the mean \pm SD and were compared using paired Student's $t$-tests. $P$ value $<0.05$ was considered as the statistical significance level.

\section{Study of in vivo heat generation after iron-dextran injection}

Two hundred $\mu \mathrm{L}$ of NCI-H460-luc2 $\left(5 \times 10^{6}\right)$ cells in saline solution was injected into the subcutaneous region of female nude mice with a BALB/c genetic background to generate cancer-bearing mice. Cancer growth was monitored daily. When the tumors reached a size of $\sim 100 \mathrm{~mm}^{3}$, the 24 cancer-bearing mice were grouped into 4 groups with 6 mice per group. Two cancer-bearing mice groups were i.v. injected with irondextran solution $(10 \mathrm{mg} / \mathrm{kg} /$ day $)$, and the other two groups were i.v. injected with dextran solution $(10 \mathrm{mg} /$ $\mathrm{kg} /$ day). Two mouse groups of iron-dextran and dextran were used for local hyperthermia experiment and the other two groups were used for whole-body hyperthermia. The iron-dextran in vivo condition protocol was followed as previously described [11]. The detailed protocol for iron-dextran in vitro condition and temperature measurement method on cancer and normal subcutaneous areas are described Additional file 1. 


\section{Organ distribution of iron ion concentration after iron-dextran injection}

The organs (liver, heart, lung, kidney, muscle, brain, stomach, and tumor) distribution of iron ion were measured in tumor-bearing mice. A small piece $(\sim 1 \mathrm{~g})$ of each organ including tumor was isolated from the cancer-bearing mice after injections of dextran or irondextran. The iron ion concentrations of each organ were measured by an Inductively Coupled Plasma Mass Spectrometry (Varian 800-MS; Varian Medical Systems, Palo Alto, CA, USA). The preprocessing protocol for measurement on iron ion concentration was followed as previously described [11].

\section{The anti-tumor efficacy of iron-dextran in vivo}

The anti-tumor efficacy of iron-dextran in vivo protocol was followed as previously described [11]. An IVIS imaging system (PerkinElmer, Waltham, MA, USA) was used to generate a bioluminescent image. The detailed preprocessing protocol for measuring in vivo imaging is described Additional file 1.

\section{Histology of tumor tissue}

The tumor tissue specimens from the cancer-bearing mice of the experimental groups were surgically isolated. After tumor tissues were fixed at $10 \%$ neutralbuffered formalin (Sigma Aldrich, St. Louis, MO, USA), followed by serial sectsion $(5 \mu \mathrm{m})$ of tumor tissue specimens were cut and stained with hematoxyline and eosin $(H \& E)$ as previously described [13, 14]. The stained tissue images were scanned by using a slide scanner, Aperio Scanscope FL (Leica Biosystems, Wetzlar, Germany), and the scanned image was processed by ImageScope Software (Aperio Technologies, Vista, CA, USA).

\section{Results}

\section{Iron-dextran generated dielectric heat in RF wave}

Iron-dextran is a complex of iron ion and dextran. Interestingly, iron ion is capable of generating heat by RF waves and dextran is capable of targeting tumor tissues. It would be logical to speculate whether iron-dextran can be selectively delivered to cancer cells where it could generate dielectric heat upon irradiation with RF waves. Therefore, we performed a heat induction experiment on iron-dextran solution in a RF wave along with dextran solution as a control. As expected, the iron-dextran solution elevated the temperature of the solution greater than the dextran solution after irradiation of $13.56 \mathrm{MHz}$ RF wave (Fig. 1a). This result demonstrated that the iron ion of iron-dextran reacted with $\mathrm{RF}$ to generate dielectric heat.
In addition to generation of dielectric heat, dextran is known to have cancer-targeting characteristics [15]. In order to test whether iron-dextran can selectively deliver iron to cancer cells, cancer and normal cells were exposed to iron-dextran, and heat generation was compared upon treating with RF thermotherapy (Fig. 1a). The exposure of cancer cells to a medium containing iron-dextran boosted temperature elevation in a dose-dependent manner in contrast to normal primary cells (Fig. 1b, Additional file 1: Fig. S1). Overall, these experiments indicated that cancer cells selectively accumulated iron ion after iron-dextran treatment to react with RF wave and induce dielectric heat more strongly. This result suggests that iron-dextran could be a possible candidate for a safe and effective thermosensitizer for RF thermotherapy.

The selectively accumulated iron ion in cancer cells reacted with a $13.56 \mathrm{MHz} \mathrm{RF}$ In order to evaluate irondextran's potential as a thermosensitizer for RF thermotherapy, we tested whether repeated injections of iron-dextran can selectively accumulate iron ion in cancer cells. Continuous injections of iron-dextran into cancer-bearing mice resulted in a gradually increasing concentration of iron ion in cancer cells in a dose dependent manner (Additional file 1: Fig. S2). We also measured the distribution of iron ion in cancer-bearing mice before and after i.v. injection of iron-dextran and dextran, respectively. As expected, iron ion successfully accumulated in tumor tissues after i.v. injection of irondextran compared to dextran injection (Additional file 1: Table S2). These results clearly demonstrate that irondextran is capable of selectively accumulating in tumor tissues when injected into tumor-bearing mice. After the confirmation of iron-dextran's selective accumulation, we conducted experiments using a tumor-xenograft model to confirm whether selective iron ion accumulation in the cancer cells will boost temperature elevation during RF thermotherapy. Various frequencies of RF have currently being adopted in modern RF thermotherapies, and irondextran should affect cancers similarly in each of RF frequencies [16]. In this work, we chose a RF hyperthermal machine using $13.56 \mathrm{MHz}$ RF which frequency is most widely used currently [17]. Local $13.56 \mathrm{MHz}$ RF hyperthermia after i.v. injection of iron-dextran increased the temperature of the cancer tissue of cancer-bearing mice by $9.96{ }^{\circ} \mathrm{C}$, compared to $4.2{ }^{\circ} \mathrm{C}$ in the cancer tissue of the dextran injection group (the control group) (Fig. 2c, Additional file 1: Fig. S3).

Since in vitro experiment showed a positive correlation between the iron ion concentration and temperature elevation under RF hyperthermia (Fig. 1b), the in vivo effect of iron ion accumulation in cancer cells under RF hyperthermia was further investigated. Figure 3 shows that cancer 

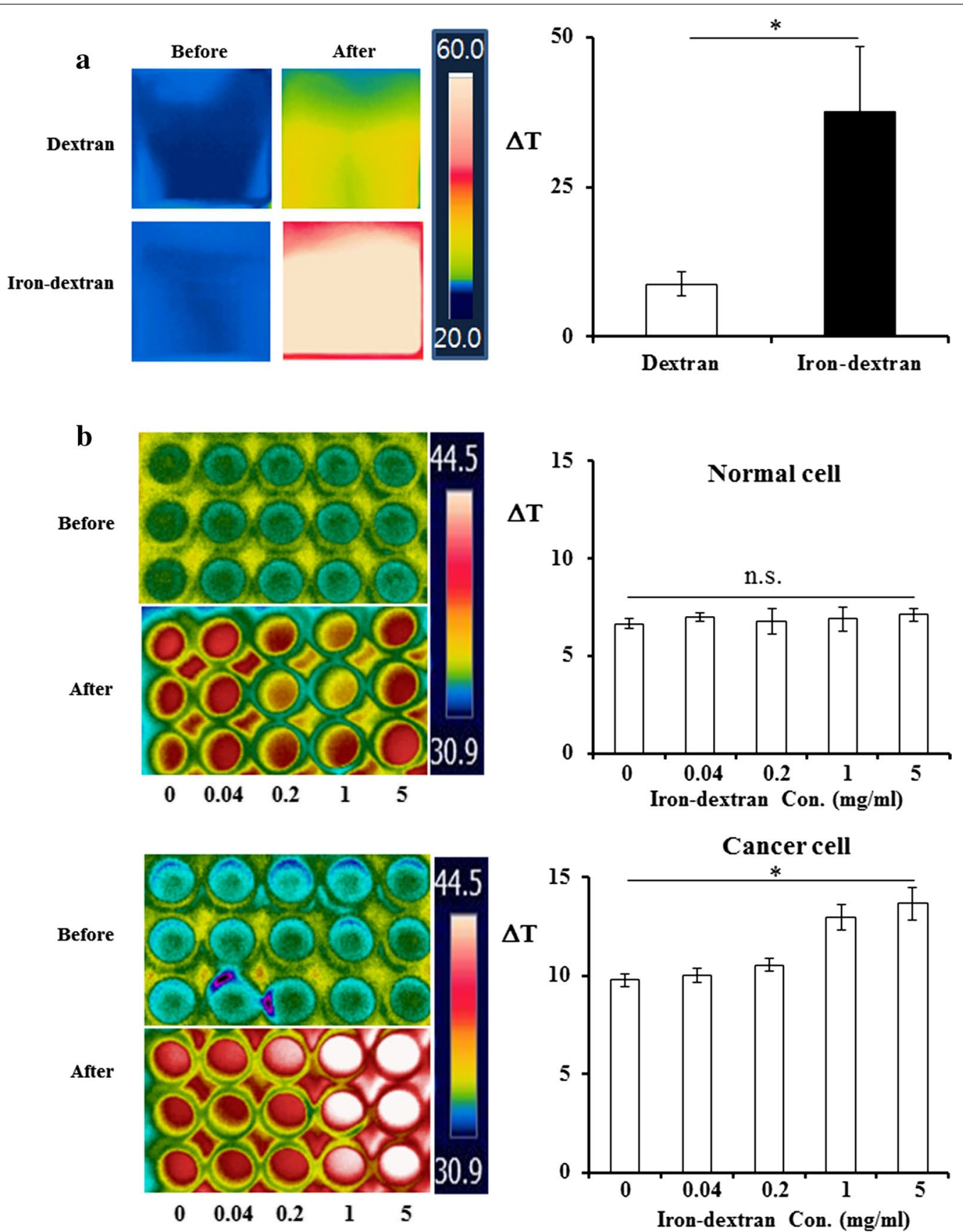

Fig. 1 Iron-dextran induced dielectric heat in the $13.56 \mathrm{MHz}$ RF wave to boost temperature elevation. a The iron-dextran generated heating upon RF hyperthermia and the temperature was elevated significantly compared to iron-free dextran solution. $\mathbf{b}$ Exposure of human lung cancer $\mathrm{NCl}-\mathrm{H} 460$ cells to iron-dextran boosted temperature elevation in a dose-dependent manner while this effect was not observed in normal cells. The values represent the mean $\pm S D(n=6)$. A paired Student's t-test was used for the statistical analysis; ${ }^{*} p<0.05$

tissues reacted greater in $13.56 \mathrm{MHz}$ RF hyperthermia as iron ion concentration was increased by repeated injections of iron-dextran into the mice. The local hyperthermia on the mice after the $8^{\text {th }}$ injection resulted in a temperature increase of $15.3{ }^{\circ} \mathrm{C}$ in the cancer tissue, while the temperature only increased by $5.2{ }^{\circ} \mathrm{C}$ in the cancer tissue of the dextran-treated group (Fig. 3a). Similar to local hyperthermia, a whole-body hyperthermia on the cancerbearing mice after $8^{\text {th }}$ injection of iron-dextran elevated temperature by $3.1{ }^{\circ} \mathrm{C}$ in the cancer tissue, while only $1.2^{\circ} \mathrm{C}$ 


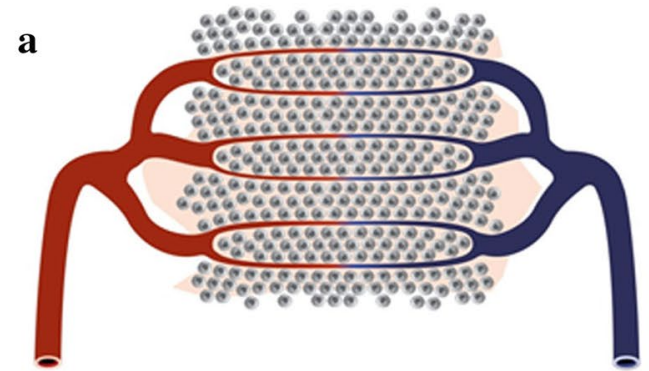

A schematic diagram of normal tissue

b

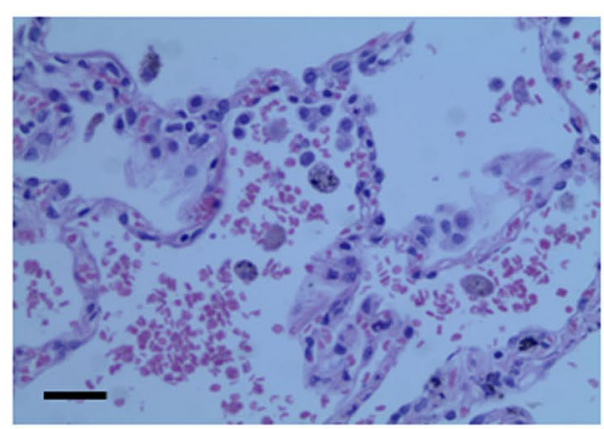

Normal lung tissue

c

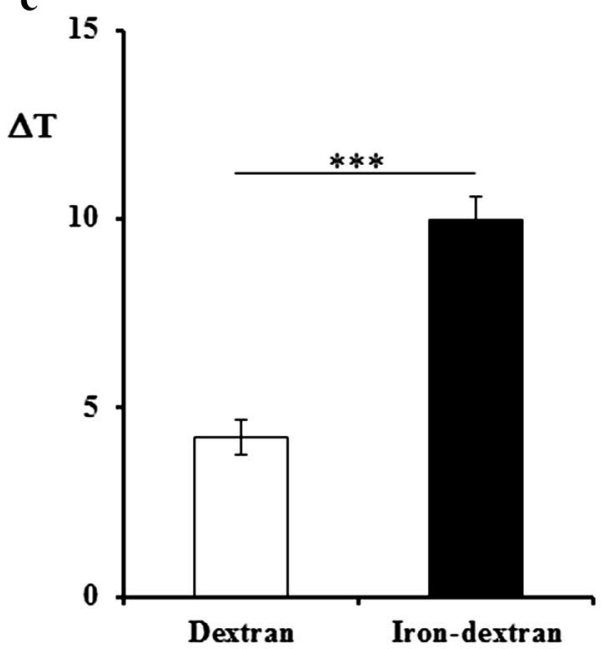

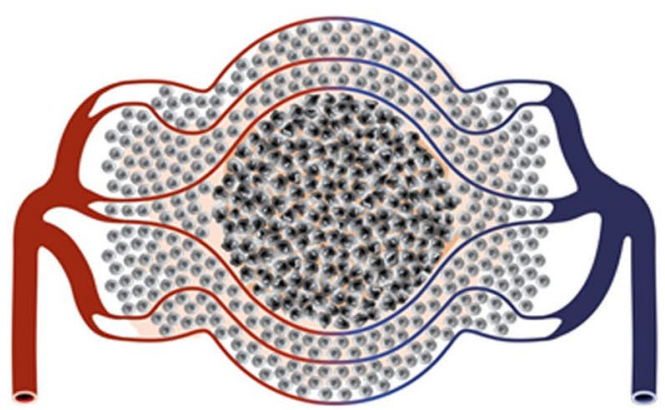

A schematic diagram of lung cancer tissue

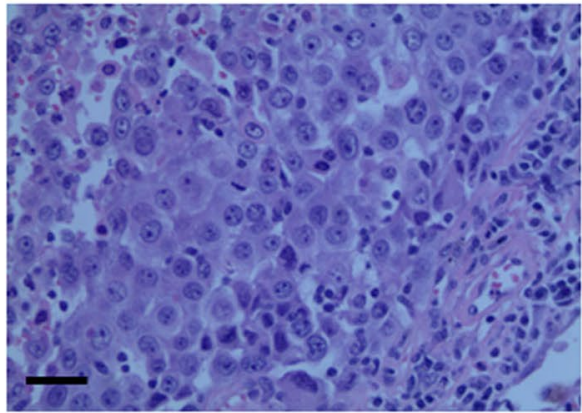

Lung cancer tissue
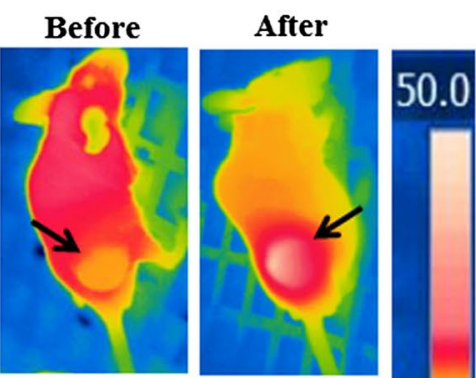

Iron-dextran
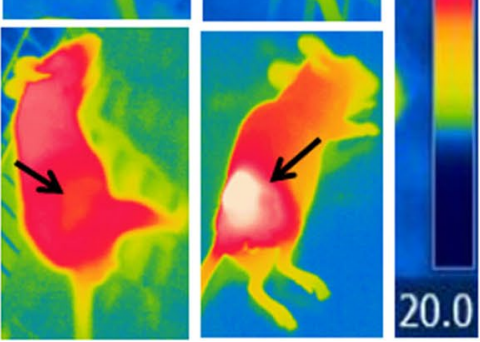

Fig. 2 Iron-dextran boosted temperature elevation in cancer-bearing mice upon $13.56 \mathrm{MHz}$ RF hyperthermia. a Schematic drawing of vasculature in cancer and normal tissues. The excessive heat in the cancer tissues is difficult to be diffused into other parts of body via blood flow, resulting cancer tissues more vulnerable to hyperthermic oncotherapy. $\mathbf{b}$ H\&E staining of cancer tissue showed the abnormal chaotic vasculature with active angiogenesis and slowed blood flow. Scale bar, $100 \mu \mathrm{m}$. c The temperature elevation in tumor tissue was dramatically boosted by local hyperthermia using $13.56 \mathrm{MHz}$ RF wave with iron-dextran but not with dextran. Data represent the mean \pm SD $(n=6)$. Asterisk indicates statistical significance as analyzed by a paired Student's t-test; ${ }^{* * *} p<0.001$ with the division

increased in the cancer tissue of the dextran-treated group (Fig. 3b, Additional file 1: Table S3). The cancer-selective accumulation of iron ion and subsequent tumor-selective heating by RF hyperthermia became more evident when the temperature change of normal subcutaneous tissue was compared to that of tumor tissue (Additional file 1: Fig. S4, Fig. S5). Temperature change over repeated injection of iron-dextran in local hyperthermia has increased up 

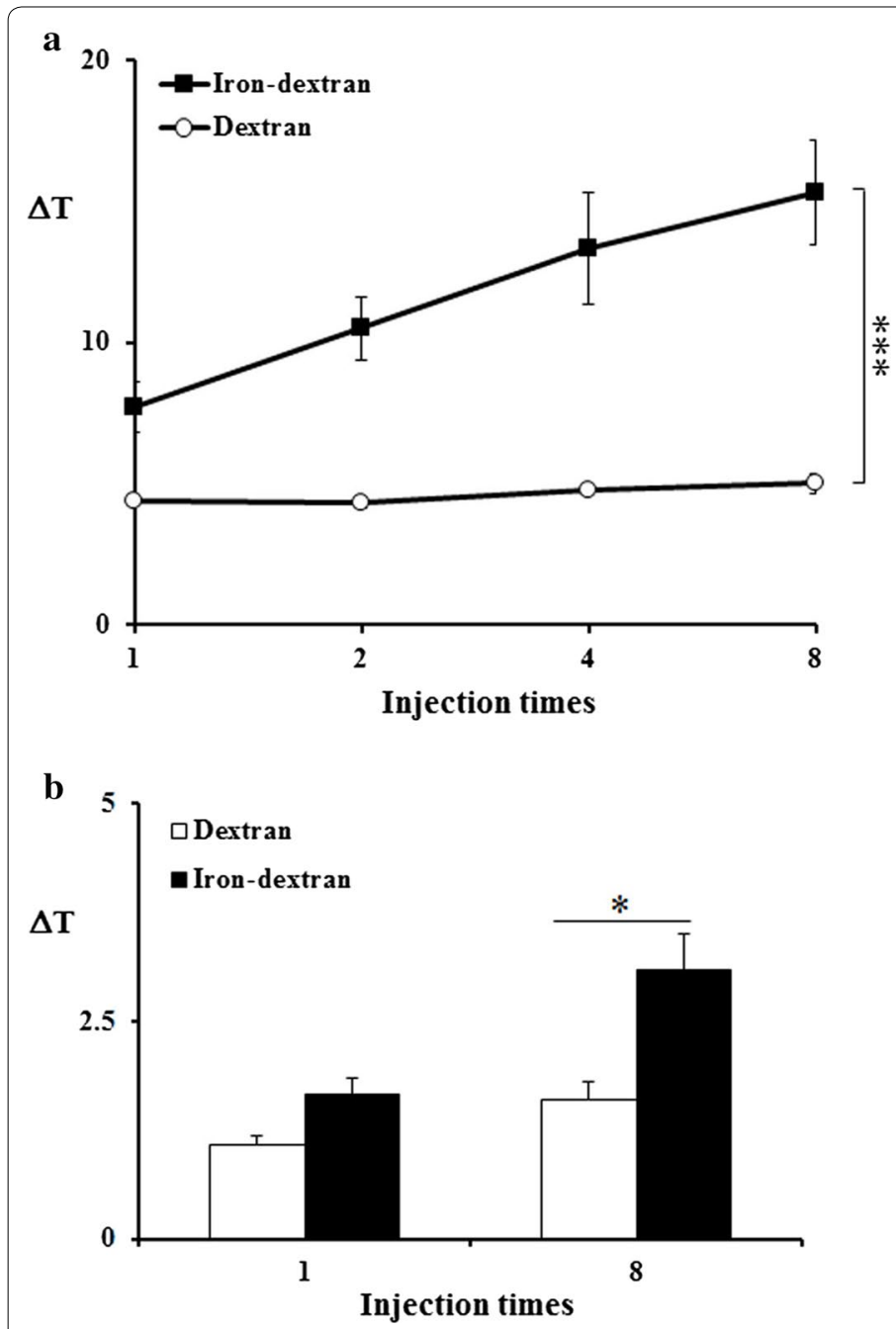

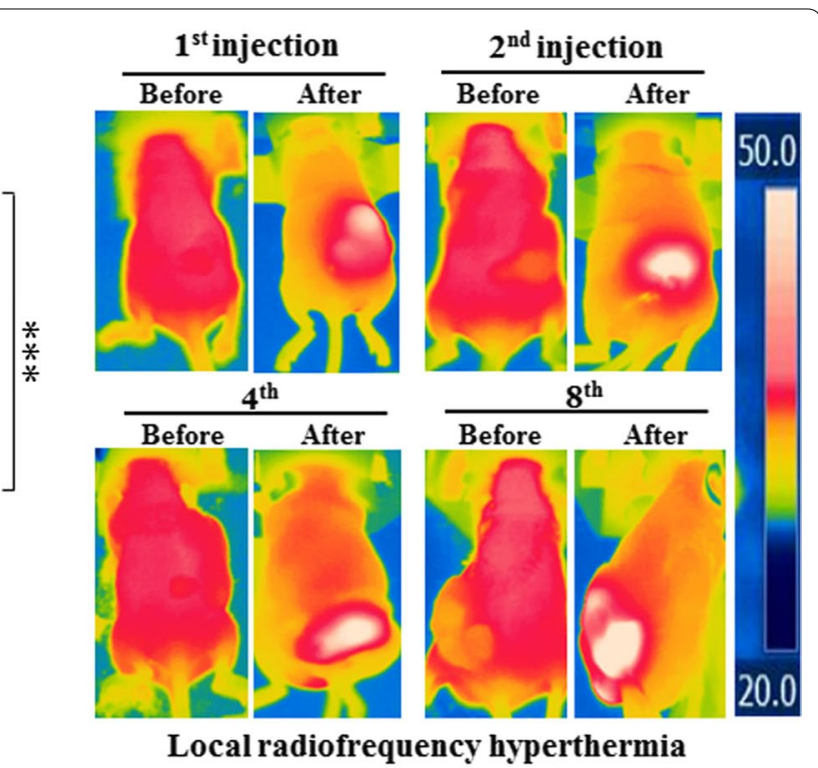

Local radiofrequency hyperthermia

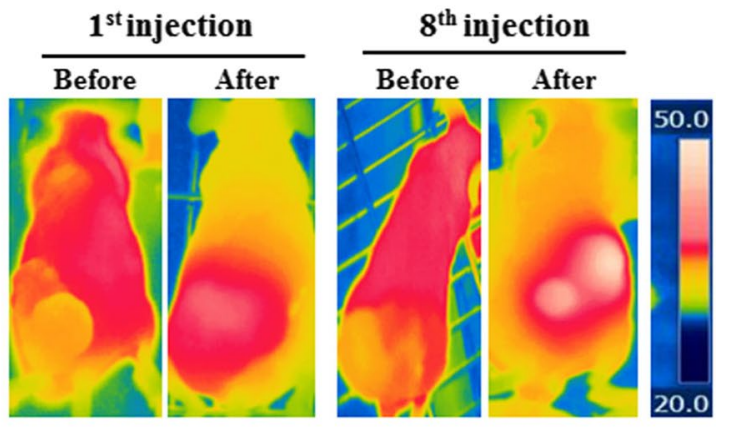

Whole-body radiofrequency hyperthermia

Fig. 3 The tumor tissues became more reactive to $13.56 \mathrm{MHz}$ RF hyperthermia as i.v. injection of iron-dextran. a The repeated i.v. injection of iron-dextran to cancer-bearing mice boosted temperature elevation greater in a local $13.56 \mathrm{MHz}$ RF hyperthermia as iron ion concentration was increased by repeated injections. b The repeated i.v. injection of iron-dextran boosted temperature elevation in a whole-body $13.56 \mathrm{MHz}$ RF hyperthermia as iron ion accumulated. The values represent the mean \pm SD $(n=6)$. A paired Student's t-test was used for the statistical analysis; ${ }^{*} p<0.05,{ }^{* * *} p<0.001$

to $15.3{ }^{\circ} \mathrm{C}$ in the cancer tissue but only to $3.4{ }^{\circ} \mathrm{C}$ in normal subcutaneous tissues (Additional file 1: Fig. S5). In accordance to the local hyperthermia, whole body hyperthermia after repeated injection of iron-dextran also resulted in tumor-selective temperature elevation (Additional file 1: Fig. S6, Fig. S7). These results clearly indicate that the selective accumulation of iron ion in the cancer cells leads to selective dielectric heating in cancer tissues in RF hyperthermia.

\section{Application of iron-dextran as a thermosensitizer in RF hyperthermia}

Since iron-dextran is able to induce tumor-selective temperature elevation under RF hyperthermia through selective accumulation of iron ion in cancer cells, we conducted animal experiments on the oncotherapeutic efficacy of iron-dextran as a thermosensitizer under RF hyperthermia. The cancer-bearing mice were treated every 3 days with local hyperthermia with $13.56 \mathrm{MHz}$ RF after i.v. injection of $10 \mathrm{mg} / \mathrm{kg} /$ day iron-dextran as a thermosensitizer. The local RF hyperthermia after irondextran injection dramatically reduced the size of cancer tissue as thermotherapy continued and completely eradicated cancer after 5 weeks of treatment (Fig. 4a, b). The overall anticancer efficacy of RF hyperthermia with iron-dextran thermosensitizer was much greater than the oncotherapeutic efficacy of paclitaxel, a standard pharmaceutical drug for chemotherapy (Fig. 4a, b). In 

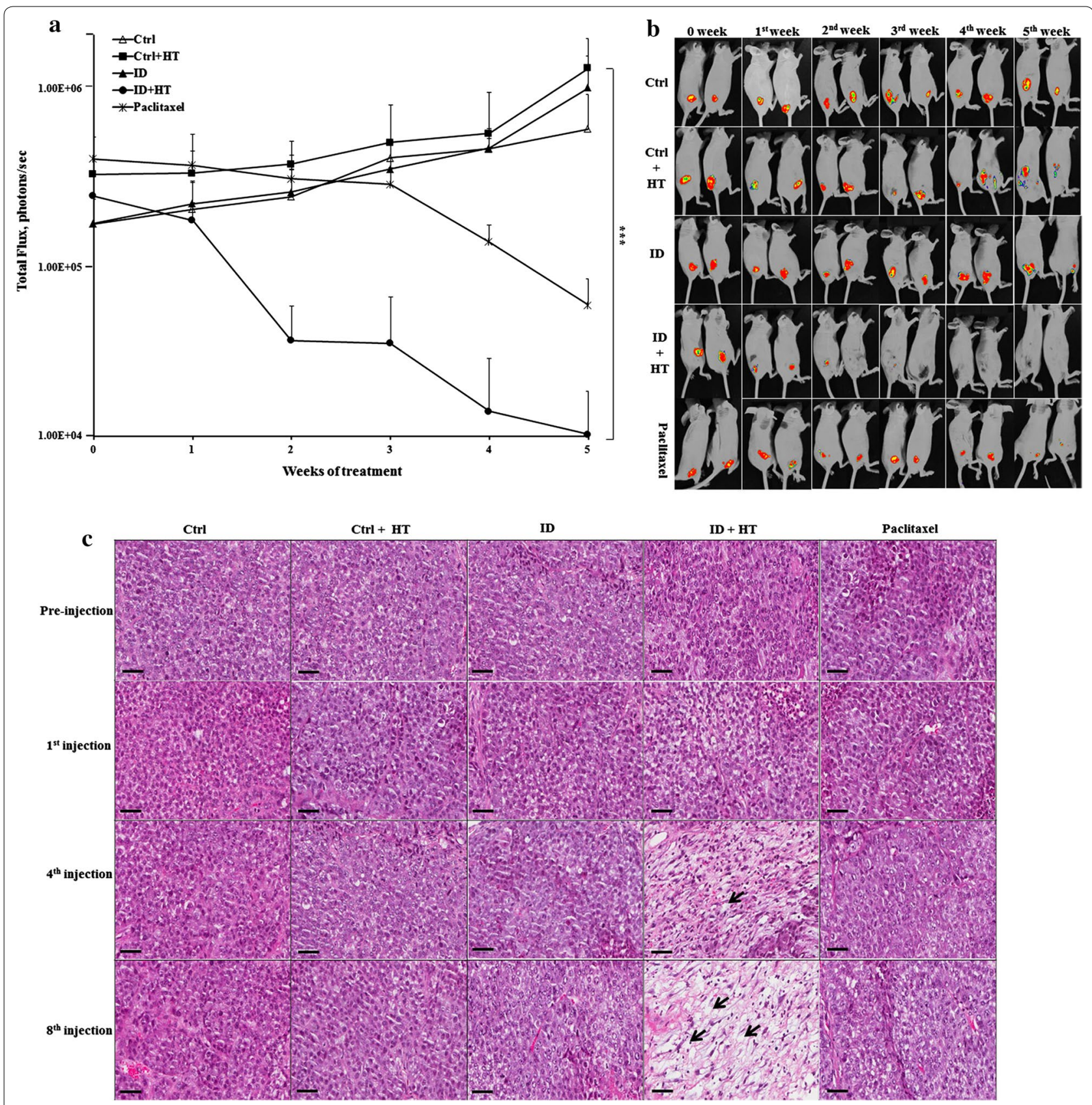

Fig. 4 The oncotherapeutic efficacy of iron-dextran as a thermosensitizer in a local $13.56 \mathrm{MHz}$ RF hyperthermia. a The in vivo evaluation results of oncotherapeutic efficacy of iron-dextran as a thermosensitizer in the local $13.56 \mathrm{MHz}$ RF hyperthermia in a tumor-xenografted mouse model. $\mathbf{b}$ The representative in vivo bioimage of the tumor-xenografted mice to evaluate the oncotherapeutic efficacy of iron-dextran as a thermosensitizer in $13.56 \mathrm{MHz}$ RF hyperthermia. c The representative histological images of the cancer tissues of the tumor-xenografted mice after each treatment. The abbreviation in this figure, $\mathrm{Ctrl}$; control mice with dextran injection, $\mathrm{Ctrl}+\mathrm{HT}$; control mice with dextran injection followed by local treatment of $13.56 \mathrm{MHz}$ RF hyperthermia, ID; mice with iron-dextran injection only, ID + HT; mice with iron-dextran injection as a thermosensitizer followed by local treatment of $13.56 \mathrm{MHz}$ RF hyperthermia, Paclitaxel; mice in which equivalent dose of paclitaxel was injected as a chemotherapeutic agent without RF hyperthermia treatment. Necrosis of tumor tissue is indicated by an arrow. The values represent the mean $\pm \operatorname{standard}$ deviation $(n=6)$. A paired Student's t-test was used for the statistical analysis; ${ }^{* *} p<0.001$. Scale bar, $50 \mu \mathrm{m}$ 
accordance with the dramatic efficacy in tumor abolishment, H\&E staining revealed a typical display of massive cell death by necrosis on the tumor tissues after the therapy (Fig. 4c). Common necrosis features such as swollen cytoplasm, early loss of membrane integrity, and disintegration (swelling) of organelles were easily identifiable in cancer cells of the iron-dextran-treated group after multiple treatments (Fig. 4c). In contrast, such signs of necrosis were not observed in any other experimental groups (Fig. 4c). These results indicate that the RF thermotherapy using iron-dextran as a thermosensitizer caused massive necrosis of cancer cells, suggesting that iron-dextran is an ideal thermosensitizer for boosting the oncotherapeutic efficacy of RF hyperthermia.

\section{Discussion}

RF thermotherapy is a hyperthermal therapy that is mostly used as a subsidiary treatment to standard cancer therapies in modern medicine. Even though RF thermotherapy is used as a subsidiary treatment, RF thermotherapy is getting popularity in present clinics because of its safety and a modest efficacy. However, ironically, the main obstacle of RF thermotherapy in cancer treatments is the limitation on temperature elevation. The RF thermotherapy cannot selectively elevate the temperature in cancer tissues, limiting elevation of the temperature of cancer tissues to the safe range of $40-42{ }^{\circ} \mathrm{C}$ in which surrounding normal tissues are not damaged. Obviously, such a range of $40-42{ }^{\circ} \mathrm{C}$ temperature is not enough to kill cancer cells, instead only causing a few cancer cells to undergo apoptosis. The range of elevated temperature might explain a limit on oncotherapeutic efficacy of current RF thermotherapy.

RF thermotherapy kills cancer cells through apoptosis. It should be noted that this is a different kind of cell death than necrosis which was observed when i.v. injection of iron-dextran was used as a thermosensitizer in RF thermotherapy (Fig. 4c). Necrosis is the premature cell death, which is caused by infection, toxins, trauma, or high heat. Unlike apoptosis, which is triggered by gentle external factor, necrosis is triggered by harsh external factors such as high heat. RF thermotherapy alone elevates the temperature of cancer cells up to $40-42{ }^{\circ} \mathrm{C}$ at which only a few cells die through apoptosis. Considering the range of elevated temperature of current RF thermotherapy, it is unrealistic to expect that the current RF thermotherapy is a reasonable choice for an efficient cancer treatment. This work shows that the application of iron-dextran as a thermosensitizer in RF thermotherapy could selectively elevate temperature in cancer tissues up to $47^{\circ} \mathrm{C}$ with minimal damage to normal tissues, which cannot be achieved by current RF thermotherapy. It should be noted that, however, IR thermography measurement used in this study has a limit in representing the defined temperatures in heterogeneous curved tumor tissue although it is one of most widely used method for thermotherapy. Consequently, it should be considered as the mean numbers representing 25 spots randomly chosen by program in tumor and normal tissues for comparative analysis.

Dextran is a complex branched polysaccharide made of various lengths of glucose residues, which is manufactured from bacterial fermentation [18]. Because of its higher molecular weight, biocompatibility, biodegradability and hydrophilic nature, dextran has been used in various pharmaceutical purposes such as plasma volume expanders, anticoagulants, drug carriers, a targeting moiety of nanoparticles for cancer, etc. [10, 19]. Especially, the large molecular weight as well as other physical characteristics of dextran made it ideal for tumor-targeting through enhanced and the enhanced permeability and retention effect. Also, glucose itself tends to be drawn by cancerous tissue. Because of these two reasons, dextran is used as one of the favored targeting moieties of current nanoparticles for cancer $[20,21]$. In an agreement with those previously known feature of dextran, our experimental data clearly confirmed the cancer-targeting nature of dextran. Because of the excellent cancer-targeting ability, injection of iron-dextran make iron ion targeted into cancer, which resulted in selective accumulation of iron ion in the cancer tissue (Additional file 1: Table S2, Fig. S2).

In this work, we show that i.v. injection of iron-dextran into cancer-bearing mice selectively delivers iron ion to cancer cells, resulting cancer-specific accumulation of iron ion. The accumulated iron ions dramatically boost the cancer-specific dielectric heating in RF thermotherapy, selectively elevating the temperature of cancer cells strong enough to cause necrosis without damaging normal tissues so as to eradicate cancer. Iron-dextran is one of the safest drugs currently used for treating anemia [22]. These characteristics make iron-dextran an ideal choice for RF thermotherapy as a thermosensitizer. Considering current oncotherapeutic efficacy of RF hyperthermia even without a thermosensitizer as well as the oncotherapeutic efficacy showed in this work, the development of iron-dextran as a thermosensitizer in RF hyperthermia should be a major progress in cancer treatment. 


\section{Additional file}

Additional file 1: Table S1. Electromagnetic waves widely used in modern medicine. Table S2. Distribution of ferric ion in each organ after single i.v. injection of iron-dextraninto tumor-bearing mice. Table S3. Temperature elevation of each organ after eight i.v. injection of iron-dextran into tumor-bearing mice. Figure $\mathbf{S 1}$. The effect of iron-dextran exposure to cancer and normal primary cells. Figure S2. The effect of repeated i.v. injectionof iron-dextran into tumor-bearing mice. Figure S3. Application of iron-dextran as a thermosensitizer in a local $13.56 \mathrm{MHz}$ radiofrequency hyperthermia boosted cancer-selective temperature elevation in tumorbearing animals. Figure S4. The positive co-relationship between the cancer-selective temperature elevation and ferric ion accumulation in a local $13.56 \mathrm{MHz}$ radiofrequency hyperthermia. Figure S5. The selective temperature elevations of cancer tissue by a local $13.56 \mathrm{MHz}$ radiofrequency hyperthermia were positively correlated with iron ion accumulations. Figure S6. The positive correlation between the cancer-selective temperature elevation and ferric ion accumulation in a whole-body 13.56 $\mathrm{MHz}$ radiofrequency hyperthermia. Figure $\mathbf{S 7}$. The selective temperature elevation of cancer tissue by a whole body $13.56 \mathrm{MHz}$ radiofrequency hyperthermia were positively correlated with iron ion accumulations by repeated injections of iron-dextran.

\section{Authors' contributions}

HJC and STH analyzed the data and wrote the paper. STH provided technical assistance to HJC and helped with the data collection. HJK edited the manuscript. STH supervised the work. STH and HJC revised and edited the manuscript and supervised the work. All authors read and approved the final manuscript.

\section{Author details}

1 Department of Biomedical Sciences and Institute for Medical Science, Chonbuk National University Medical School, Jeonju, Chonbuk, South Korea. ${ }^{2}$ JINIS BDRD Institute, JINIS Biopharmaceuticals Co., 913 Gwahak-Ro, Bongdong, Wanju, Chonbuk, South Korea.

\section{Acknowledgements}

This research was supported by the BDRD Research Fund from JINIS Biopharmaceutical Co., Republic of Korea.

\section{Competing interests}

The authors declare that they have no competing interests.

\section{Availability of data and materials}

All data is available in the main text or the supplementary materials.

\section{Publisher's Note}

Springer Nature remains neutral with regard to jurisdictional claims in published maps and institutional affiliations.

\section{Received: 27 March 2019 Accepted: 02 April 2019}

Published online: 16 April 2019

\section{References}

1. Denekamp J (1993) Angiogenesis, neovascular proliferation and vascular pathophysiology as targets for cancer therapy. Br J Radiol 66:181-196
2. Busch T, Bärtsch P, Pappert D, Grünig E, Hildebrandt W, Elser H, Falke KJ (2001) E.R.Hypoxia decreases exhaled nitric oxide in mountaineers susceptible to high-altitude pulmonary edema. Am J Respir Crit Care Med 163:368-373

3. Horsman MR, Vaupel P (2016) Pathophysiological basis for the formation of the tumor microenvironment. Front Oncol 6:66

4. Roussakow S (2013) The history of hyperthermia rise and decline. Conf Papers Med 2013:140

5. Anselmo AC, Mitragotri S (2015) A review of clinical translation of inorganic nanoparticles. AAPS J 17:1041-1054

6. Rana S, Kalaichelvan PT (2013) Ecotoxicity of nanoparticles. ISRN Toxicol 2013:1-11

7. Bae KH, Park M, Do MJ, Lee N, Ryu JH, Kim GW, Kim CG, Park TG, Hyeon T (2012) Chitosan oligosaccharide-stabilized ferrimagnetic iron oxide nanocubes for magnetically modulated cancer hyperthermia. ACS Nano 6:5266-5273

8. Chan DCF, Kirpotin DB, Bunn PA Jr (1993) Synthesis and evaluation of colloidal magnetic iron oxides for the site-specific radiofrequency-induced hyperthermia of cancer. J Magn Magn Mater 122:374-378

9. Wolf M, Koch TA, Bregman DB (2013) Effects of iron deficiency anemia and its treatment on fibroblast growth factor 23 and phosphate homeostasis in women: FGF23 in iron deficiency. J Bone Miner Res 28:1793-1803

10. Li B, Wang Q, Wang X, Wang C, Jiang X (2013) Preparation, drug release and cellular uptake of doxorubicin-loaded dextran-b-poly ( $\boldsymbol{\varepsilon}$-caprolactone) nanoparticles. Carbohydr Polym 93:430-437

11. Chung HJ, Lee HK, Kwon KB, Kim HJ, Hong ST (2018) Transferrin as a thermosensitizer in radiofrequency hyperthermia for cancer treatment. Sci Rep 8:13505

12. Baek SH, Chung HJ, Lee HK, D'Souza R, Jeon Y, Kim HJ, Kweon SJ, Hong ST (2014) Treatment of obesity with the resveratrol-enriched rice DJ526. Sci Rep 4:3879

13. Chung HJ, Sharma SP, Kim HJ, Baek SH, Hong ST (2016) The resveratrolenriched rice DJ526 boosts motor coordination and physical strength. Sci Rep 6:23958

14. Chung HJ, Chen $X$, Yu Y, Lee HK, Song CH, Choe H, Lee S, Kim HJ, Hong ST (2018) A critical role of hepatitis B virus polymerase in cirrhosis, hepatocellular carcinoma, and steatosis. FEBS Open Bio 8:130-145

15. Dong C, Tate JA, Kett WC, Batra J, Demidenko E, Lewis LD, Hoopes PJ, Gerngross TU, Griswold E (2015) Tumor cell targeting by iron oxide nanoparticles is dominated by different factors in vitro versus in vivo. PLoS ONE 10:e0115636

16. Habash RWY, Bansal R, Krewski D, Alhafid HT (2006) Thermal therapy, part 1: an introduction to thermal therapy. Crit Rev Biomed Eng 34:459-489

17. Collins CB, McCoy RS, Ackerson BJ, Collinsc GJ, Ackerson CJ (2014) Radiofrequency heating pathways for gold nanoparticles. Nanoscale 6:8459-8472

18. Staat RH, Gawronski TH, Schachtele CF (1973) Detection and preliminary studies on dextranase-producing microorganisms from human dental plaque. Infect Immun 8:1009-1016

19. Angelopoulou A, Efthimiadou EK, Kordas G (2012) Dextran modified pH sensitive silica hydro-xerogels as promising drug delivery scaffolds. Mater Lett 74:50-53

20. Brannon-Peppas L, Blanchette JO (2012) Nanoparticle and targeted systems for cancer therapy. Adv Drug Deliv Rev 64:206-212

21. Steichen SD, Caldorera-Moore M, Peppas NA (2013) A review of current nanoparticle and targeting moieties for the delivery of cancer therapeutics. Eur Pharm Sci 48:416-427

22. Kalaivani K (2013) Use of intravenous iron sucrose for treatment of anaemia in pregnancy. Indian J Med Res 138:16-17 